\title{
EVERY CLOSED ORIENTABLE 3-MANIFOLD IS A 3-FOLD BRANCHED COVERING SPACE OF $S^{3}$
}

\author{
BY HUGH M. HILDEN ${ }^{1}$
}

Communicated by William Browder, March 20, 1974

Let $p: M \rightarrow N$ be a nondegenerate simplicial map between compact triangulated manifolds of the same dimension $n$. This is a branched covering space if the restriction of $p$, called $\tilde{p}$, gives a covering space map $\tilde{p}: M-$ ( $n-2$ skeleton) $\rightarrow N-(n-2$ skeleton). The set of points $x$ in $M$ such that $p$ does not map any neighborhood of $x$ homeomorphically into $N$ is called the branch cover $B$. The $(n-2)$-dimensional set $p(B)$ is called the branch set. J. W. Alexander asserted the following theorem [1] .

AleXANDER'S ThEOREM. Every closed orientable 3-manifold is a branched covering space of $S^{3}$ with the branch set a link in $S^{3}$.

For a proof see [5].

The purpose of this paper is to announce the following result.

THEOREM 1. Every closed orientable 3-manifold is an irregular 3-sheeted branched covering of $S^{\mathbf{3}}$ with branch set a knot.

We shall only sketch the proof of the theorem. A detailed proof will appear elsewhere.

The main part of the proof consists of constructing a certain irregular 3 fold branched covering of the 3-ball, $D^{3}$, by a handlebody of genus $g, X_{g}$, with branch set $A$, a set of $g+2$ proper arcs, and observing that for this particular branched covering any homeomorphism $\psi$ of $\partial X_{g}$ is isotopic to a homeomorphism $\phi$ that projects to a homeomorphism $\phi$ of $\partial D^{3} . \phi$ necessarily leaves the branch set $A \cap \partial D^{3}$ invariant as a set. Moreover we can choose $\phi$ and $\phi$ so that $\phi$ induces a cyclic permutation on the branch set. Let $i(i)$ be the map that identifies $X_{g}$ with $X_{g}^{\prime}\left(D^{3}\right.$ with $\left.D^{3^{\prime}}\right)$ restricted to the

AMS (MOS) subject classifications (1970). Primary 55A10, 57A10; Secondary 55A25.

Key words and phrases. Branched covering, 3-manifold, knot, link.

1 This work was supported by NSF grant GP-34059. 
boundary. Any 3-manifold of genus $g, M$, has the presentation $M=X_{g} \cup_{\phi i}$ $X_{g}^{\prime}$ for some homeomorphism $\phi$ of $\partial X_{g}$ that projects to a homeomorphism $\phi$ of $\partial D^{3}$.

$$
p \cup p^{\prime}: X_{g} \cup_{\phi i} X_{g}^{\prime} \rightarrow D^{3} \cup_{\phi \mathbf{i}} D^{3^{\prime}}=S^{3}
$$

is a branched covering space with branch set the knot $A \cup_{\phi \mathbf{i}} A^{\prime}$.

It follows from the particular construction that the inverse image of a point in the branch set consists of two points, one in the branch cover and one not in the branch cover.

J. M. Montesinos (correspondence with the author) has obtained Theorem 1 independently and simultaneously using very different methods (surgery on knots and links). He had previously obtained certain other results on threesheeted branched coverings of knots of this type [3], [4], [5]. In [2] Ralph Fox gave examples of simply connected closed 3-manifolds which were threesheeted branched coverings of knots in $S^{3}$. In [3], [4], [5] Montesinos developed methods which he used to show that certain irregular branched coverings of knots were $S^{3}$. In particular he showed that the examples of Fox were all $S^{3}$, and further that any simply connected 3-sheeted covering, of this type, of a knot in the Alexander Briggs table is $S^{3}$.

\section{BIBLIOGRAPHY}

1. J. W. Alexander, Note on Riemann spaces, Bull. Amer. Math. Soc. 26 (1920), $370-372$.

2. R. H. Fox, Construction of simply connected 3-manifolds, Topology of 3-Manifolds and Related Topics (Proc. The Univ. of Georgia Inst., 1961), Prentice-Hall, Englewood Cliffs, N. J., 1962, pp. 213-216. MR 25 \#3539.

3. J. M. Montesinos, Sobre la conjetura de Poincaré y los recubridores ramificados sobre un nudo, Tesis doctoral, Publicada en Departamento de Publicaciones de la Facultad de Ciencias de la Univ. de Madrid, 1971.

4. Reduction of the Poincaré conjecture to other geometric conjectures, Rev. Mat. Hisp.-Amer. (4) 32 (1972), 33-51. (Spanish) MR 47 \#2590.

5. Una nota a un teorema de Alexander, Rev. Mat. Hisp.-Amer. (4) 32 (1972), 167-187.

DEPARTMENT OF MATHEMATICS, UNIVERSITY OF HAWAII, HONOLULU, HAWAII 96822 